\title{
Complete second arch branchial fistula in a 3-year- old child
}

\section{Clinical history}

Patient: 3 years, male

A 3-year-old boy presented with the complaints of watery discharge from an opening in the right-hand side of neck on and off since birth. There was no history of trauma or operation.

Keywords: branchial fistula, diagnosis, oropharynx, fistulography, branchial cysts

\section{Imaging findings}

A contrast study was done by injecting iodinated contrast medium through the external opening, which revealed an irregular tract with beaded appearance on right side of neck extending cranially into the right lateral wall of oropharynx. Esophagus and stomach too showed the swallowed contrast, confirming the diagnosis of complete second arch branchial fistula.

\section{Discussion}

Branchial apparatus consists of 5 mesodermal arches separated by invaginations of the ectoderm called as clefts. Anomalies of the branchial apparatus include branchial cysts, sinuses and fistulas. ${ }^{1,2}$ Branchial anomalies are common congenital lesions of the neck, of which second branchial complex anomalies are most common, accounting for about $95 \%$ of these lesions. A complete branchial fistula with 2 openings is rare. ${ }^{3}$ Branchial cysts are thought to occur due to entrapped remnants of branchial cleft or sinuses; branchial sinuses from entrapped remnants of cleft of pouches; and branchial fistulae occur from persistence of both pouch and cleft. ${ }^{4}$ The fistulous tract in second branchial fistula extends deep to the platysma, along the carotid sheath, and passes between carotid arteries bifurcation. Cranially, opening of the tract lies in the lateral wall of oropharynx at the region of tonsillar fossa. ${ }^{4}$ Second arch branchial fistula can be unilateral or bilateral. In case of unilateral, the right side preponderance is described. ${ }^{5}$ Fistulography and computed tomography (CT) fistulogram are the modality of choice for radiological diagnosis of second branchial arch fistula (Figure 1). ${ }^{1,3,5}$

Fistulography (performed with opacifying the fistula with contrast media) delineates the fistulous tract extending from the external cutaneous opening at the lateral neck to the tonsillar fossa. ${ }^{1,2} \mathrm{CT}$ fistulogram with reformatted images demonstrates the fistulous tract as well as its relationship with important structures of the neck. ${ }^{1}$ Ultrasound and magnetic resonance imaging (MRI) has less diagnostic value in the diagnosis of fistulous tract, but more advantageous in the diagnosis of branchial arch cysts. ${ }^{1,5}$ Second arch branchial fistula are usually diagnosed in infancy or childhood with patient presenting with the complaints of watery or purulent discharge from an opening at the anterior aspect of lower third of the neck. ${ }^{3}$ The treatment of choice of second branchial arch fistula is complete surgical excision of the fistulous tract. ${ }^{1,2,4}$ Complete second arch branchial fistula is congenital anomaly of neck in the paediatric age group. The incidence of branchial fistula is less as compared to branchial cysts and sinuses. Fistulography (performed with opacifying the fistula with contrast media) is the best radiological diagnostic tool for pre-operative
Volume 6 Issue $6-2019$

Manmohan Bir Shrestha, Bishwajit Bhowmik, Mahesh Chaudhary, Biva Chaudhary, Sharzia Asma-Ul Hosna, Mahin Binte Anwar, Rajeev Shah, Saurav Choudhary

Department of Radiology and Imaging, BSMMU, Bangladesh

Correspondence: Manmohan Bir Shrestha, Department of Radiology and Imaging, BSMMU, Bangladesh, Email macbishrestha@gmail.com

Received: September 30, 2019 | Published: November 29,2019

evaluation. Complete surgical excision is performed as the treatment of choice of second branchial arch fistula (Figure 2).

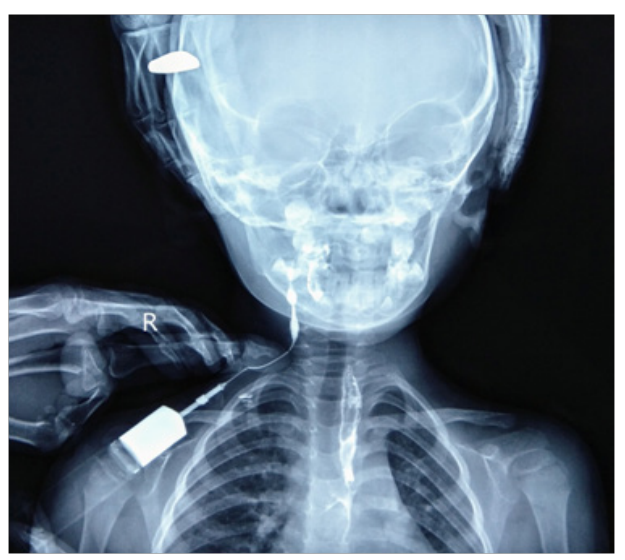

Figure I Fistulogram anteroposterior view showing the fistulous tract.

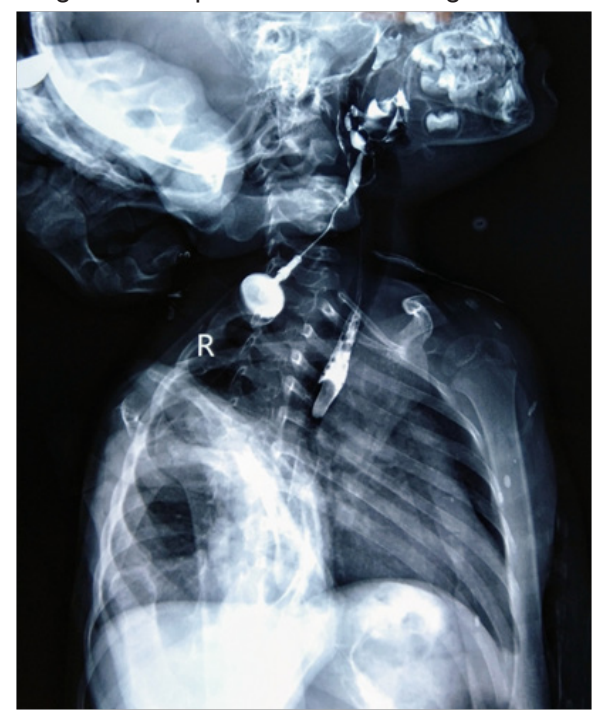

Figure 2 Fistulogram lateral view demonstrating the fistulous tract. 


\section{Acknowledgments}

None.

\section{Conflicts of interest}

Author declares that there is no conflict of interest.

\section{References}

1. Sahu S, Kumar A, Ramakrishnan TS. Branchial fistula: an imaging perspective. Med J Armed Forces India. 2011;67(3):262-264.
2. Shankar VG, Babu TA, Swami HB. A rare case of complete second arch branchial fistula in a 7-year-old child. Natl J Maxillofac Surg. 2012;3(2):226-228.

3. Adams A, Mankad K, Offiah C, et al. Branchial cleft anomalies: a pictorial review of embryological development and spectrum of imaging findings. Insights Imaging. 2015;7(1):69-76.

4. Prasad SC, Azeez A, Thada ND, et al. Branchial Anomalies: Diagnosis and Management. Int J Otolaryngol. 2014;1-9.

5. Kalra VK, Rattan KN, Yadav SPS, et al. Second Branchial Anomalies: A Study of 94 Cases. Indian J Otolaryngol Head Neck Surg. 2017;69(4):540-543. 